\title{
Analysis of Block Orders and Its Implications for Market Participation Mode of Battery Energy Storage Stations
}

\author{
Liangyuan Wang ${ }^{1}$, Shuyuan Lin ${ }^{1}$, Zongheng Xuan ${ }^{2, *}$, Wensheng Ye², Shuofan Lin ${ }^{1}$, and Shuohui Yang ${ }^{1}$ \\ ${ }^{1}$ State Grid Corporation of China, Fujian Electric Power Trading Company Limited, 350000 Fuzhou, China \\ ${ }^{2}$ South China University of Technology, School of Electric Power, 510000 Guangzhou, China
}

\begin{abstract}
Flexible block orders designed by European electricity market is a relatively perfect time-sharing electricity price and clearing mechanism. It allows members to provide bidding form of diversification and individuation according to their own characteristics. This mechanism has features of flexibility, compatibility and expansibility, and can reasonably express the trading demands of various types of market participants, which including energy storage. Therefore, it can be used to solve the problem of market participation model of energy storage. Considering the actual situation in China, block orders are suitable for the daily, weekly and monthly markets to help battery energy storage stations. So that they can obtain low-price electric energy by participating in the medium and long-term electricity market and promote the development of them.
\end{abstract}

\section{Introduction}

Chinese power industry is facing two major trends of energy transformation and power system reform [1]. In the context of energy transformation, renewable energy such as wind power and photovoltaic (PV) is increasingly permeating. The intermittent and uncertain nature of renewable energy brings great challenges to the security and stability of the power system, and it is urgent for resources with fast and flexibly such as the battery energy storage (BES) to participate in the regulation. Under the background of electric power system reform in China, many provinces and regions have successively introduced a series of reform plans and measures. The electric power market system with medium and long-term trading as the main part and spot trading as the supplement has taken shape, and the BES has more choices to participate in the electricity market than before.

At present, domestic and foreign battery energy storage stations (BESS) mainly participate in the energy, regulation and reserve market, among which the regulation market is the most important source of income. By virtue of its fast start-stop and flexible power regulation characteristics, BES is expected to improve the flexibility of power system, transfer load and reduce grid cost [2-4], and increasingly become a high-quality Automatic Generation Control (AGC) regulation resource [5-6]. In fact, the BES plays an extensive and important role on the electric energy generation, grid and user side, creating economic value for the power system [7-8] and increase the Community Surplus (CS) [9-10]. Current physical factors restricting BESS in regulation market is mainly the realization of the power balance in the process of frequency regulation. In order to solve this problem, there are usually two kinds of practice. One is that BESS and thermal power plants jointly participate in regulation market, manage the state of charge (SOC) by syndication. The second is that BESS buy or sell electric energy in realtime energy market in order to maintain the stability of its SOC, which is based on the perfection of spot market construction.

Summarizing the experience and lessons of market construction in China and other countries, electricity marketization is a process of continuous improvement and gradual progress [11-14]. At present, there are still many provinces and regions in China that have not yet run spot market. In this circumstance, how to design the market mechanism that suitable for the participation of independent BESS is a problem which needs to be urgently solved now. And the medium and long-term electricity market has mature operation experience nationwide, which is one of the modes for BESS to participate in the market and make profit. The Basic Rules for Medium and Long-term Electricity Trading issued by the National Development and Reform Commission in June 2020 clearly states that energy storage can participate in long-term electricity trading, but no specific trading scheme has been proposed.

Block order is a flexible way of bidding and clearing, which can accurately reflect the transaction demands of various market participants, and is widely used in foreign electricity markets at present. Block Orders fit the flexible charge and discharge requirements of BESS. So, this article introduces the block orders model of Nordic electricity market, analyses the clearing model of different blocks. Then, considering the actual situation of the electricity market in China, we give some implications on designing mechanism suitable for BESS to participate in the medium and long-term energy market, which can effectively motivate investment and operation of them.

\footnotetext{
* Corresponding author: epxzh@mail.scut.edu.cn
} 


\section{Market with block orders}

\subsection{Background of the market}

In the future, renewable energy is highly developed, and market with flexible combination of various types of energy have great coordination among power supply, grid, load and energy storage. This requires the design of various types of transactions and bidding forms with flexibility to meet the needs of different market participants, so that they can do some trading flexibly according to their own physical and economic characteristics.

Block Orders is an ideal schema for these problems. Block Orders is available in a wide range of markets, including medium, long-term and day-ahead markets, energy and ancillary service markets, and is a mature time-sharing bidding and clearing mechanism. Block Orders is a centralized matchmaking mechanism that is compatible with traditional time-sharing medium and long-term energy markets. In contrast to traditional markets, the markets with Block Orders add the concept of "blocks", which can have very distinct characteristics to match the characteristics of different types of products. Participants can represent their products in blocks and bid in the market to achieve their desired trading results. Block Orders was first introduced on the Nord Pool in 2004 and quickly spread to other parts of Europe because the model was so effective and easy to scale. Today, the Block Orders pattern is widely used in Central Europe, Northern Europe, the United Kingdom, and many other regions, and has developed many new types with many nice features. Block Orders has become an important time-sharing power bidding and clearing mechanism.

\subsection{Orders}

In the Nordic spot market, there are three types of orders: single hourly orders, flexible hourly orders and block orders [15]. Block orders are divided into regular block orders, flexible block orders, linked block orders, and extend linked block orders.

\subsubsection{Single hourly orders}

Flexibility is the most prominent feature of single hourly orders, which makes it account for more than half of the total volume traded in the Nordic day-ahead market [16]. Each member participating in the single hourly orders trading must declare the quantity and price of the order within the hour. Different hours are independent of each other. If users are required to participate in all-day trading, they need to declare their trading intentions for 24 periods independently. In addition, users need to consider factors such as the ramp constraints and change of the load, and take this as the basis for order declaration. Taking the electricity purchasing side as an example, if the single hourly orders declared by the electricity purchasing survey is higher than the market clearing price, the order will win the bid, otherwise it will not win the bid [17]. This mode is suitable for uncontrollable or slow climbing characteristics such as the wind power turbines, PV, the peak load of thermal power plant and non-adjustable load on the generation side.

\subsubsection{Flexible hourly orders}

Flexible hourly orders are similar with single hourly orders, requiring users to declare quantity and price, but the difference is that the period can be multiple. Orders can only win the bid once in the selected periods. Namely the same order can choose a period, declared by members of the market, to win the bid. The specific bidding period is determined according to the unified optimization of the market clearing model [18]. In other words, the winning period is the period that can create the greatest social welfare. This type of orders is mainly used in the Nordic spot market to fill the peak load gap, so for the time being only the generation side is allowed to use.

\subsubsection{Block orders}

Block Orders consist of four specific types as follows.

Regular Block: Regular block orders need to declare quantity, prices and hours as a whole block. The requirements for the declaration period are no more than 24 hours and no less than 3 hours. If the order wins the bid, means the whole block wins, otherwise, the whole block is not. This type of orders is suitable for units with high cost when adjust output frequently, such as the thermal power units of base load or nuclear power units, as well as the loads that need continuous or rigid power. They use such orders to avoid frequent participation in trading and increase transaction costs [18].

Flexible blocks: Flexible blocks are a combination of no more than three regular blocks, with a certain upper limit on the total amount of power to be declared. Similar to flexible hourly orders, flexible block orders are free to choose their starting and ending periods and can only be cleared once during these periods. The biggest difference with the flexible hourly orders is that the flexible block orders can declare different quantity and prices in different periods. This kind of orders are suitable for a certain amount of energy generation or consumption, but the time of production or use is an adjustable resource, so this kind of order offer a lot of freedom to users [19].

Linked Block: Linked blocks are made up of multiple regular blocks that prioritize. The block with higher priority is called the parent block, and the lower one is called the child block. The necessary but not sufficient condition for the child block to win the bid is that the parent block wins the bid. In order declaration, the market members shall specify the priority of each block in addition to the quantity, price and periods. A set of linked blocks must be a whole selling block or a buying block, and cannot be partly a selling block and partly a buying block [20]. It is not difficult to find out that the linked block can win part of the bid, when the parent block is making profit, the parent block will win the bid, then, the child block according to its own benefits to decide whether to win the bid. When the parent block is making loss, the parent block will not win the bid, and neither the 
child block, no matter it will make profit or loss.

Extend Linked Block: Extend linked block has expanded on the basis of linked block. Their methods of declaration are the same, but they are different in winning the bid. Extend linked block makes the whole orders win the bid when the combination of parent block and child block is profitable. The following cases are discussed: When the parent block and the child block are both making profit or loss at the same time, the whole order will win or lose the bid accordingly; When the parent block is making profit while the child block is making loss, the parent block will win the bid and the child block will not; When the parent block is making loss while the child block is making profit, the whole order can win the bid only if the combination is profitable. At this situation, we can see the child block's gain is greater than the parent block's loss.

\subsection{Market modeling}

The market clearing model considering block orders aims at maximizing social welfare and considers constraints such as coupling effect of period, quantity and price. In essence, this clearing model is a large-scale nonlinear mixed integer programming. From a mathematical point of view, since it is inconvenient to directly solve a largescale nonlinear mixed integer programming with complementary constraints in multiple periods, auxiliary variables can be added to linearize the model so that it can be solved easily with a solver.

It should be clear that block order is essentially a bid or an offer in which the clearing model represents the amount of power traded by multiplying a $0-1$ integer variable by the amount of electricity declared by a market member. In addition to adding 0-1 integer variables, it is only necessary to add the corresponding block constraints to the traditional electricity market clearing model without modifying other constraints. In this model, only the typical power balance constraints are considered. Other physical constraints are similar and will not be described. The objective function is defined as:

$$
\max \sum_{t}^{T}\left(\sum_{i}^{M} \lambda_{i}^{d} p_{i}^{d} q_{i, t}^{d}+\sum_{j}^{N} \lambda_{j}^{g} p_{j}^{g} q_{j, t}^{g}\right)
$$

In the formula, the total number of market periods is $\mathrm{T}$, the variables of the power purchasing side and the generation side are denoted by subscripts $d$ and $g$ respectively, the total number of orders is $M$ and $N$ respectively, and the quotations of corresponding orders are $p_{i}^{d}$ and $p_{j}^{g}$ respectively, both positive numbers. It is worth noting that the declared power $q_{i, t}^{d}$ of the purchasing side in the period t is positive, while the $q_{j, t}^{g}$ of the generating side is negative. In the formula, $\lambda_{i}^{d}$ and $\lambda_{j}^{g}$ are 0-1 integer variables of the corresponding orders at the purchasing side and the generation side respectively. 1 means winning the bid, 0 means not winning. For partial winning of bidding orders, it is a continuous variable within the value range $[0,1]$, and represents the proportion of the winning power.
The objective function is to maximize the total social welfare, that is, to maximize the difference between the total bid price and the total offer price.

The power balance constraints:

$$
\sum_{i}^{M} \lambda_{i}^{d} q_{i, t}^{d}+\sum_{j}^{N} \lambda_{j}^{g} q_{j, t}^{g}=0 \quad(t=1, \ldots, T)
$$

Power of both purchasing and generation sides winning the bid is balanced for each period.

The single hourly orders constraints:

$$
\begin{gathered}
\gamma_{i}^{d}\left(p_{i}^{d}-\pi_{t}\right) q_{i, t}^{d} \geq 0 \\
\gamma_{i}^{d} \geq \lambda_{i}^{d} \\
\gamma_{j}^{g}\left(p_{j}^{g}-\pi_{t}\right) q_{j, t}^{g} \geq 0 \\
\gamma_{j}^{g} \geq \lambda_{j}^{g}
\end{gathered}
$$

A market participant cannot lose money by trading. In the formula, $\pi \_\mathrm{t}$ is the clearing price of period $\mathrm{t}, \lambda_{i}^{d}$ and $\lambda_{j}^{g}$ are continuous variables with values in the range $[0,1], \gamma_{i}^{d}$ and $\gamma_{j}^{g}$ are corresponding $0-1$ integer variables, where 1 means winning the bid, while 0 means not winning.

The flexible hourly orders constraints:

$$
\sum \lambda_{i}^{d} \leq 1
$$

A flexible hourly order can only win the bid once, where $i(j)$ is the number of the single hourly orders belonging to the same flexible hour order. Each flexible hourly order shall meet the bidding constraints of the single hourly orders separately, and cannot win the bid in part.

The regular block orders constraints:

$$
\begin{aligned}
& \sum_{t=t(k)}^{T(k)} \lambda_{i}^{d}\left(p_{i}^{d}-\pi_{t}\right) q_{i, t}^{d} \geq 0 \\
& \sum_{t=t(l)}^{T(l)} \lambda_{j}^{g}\left(p_{j}^{g}-\pi_{t}\right) q_{j, t}^{g} \geq 0
\end{aligned}
$$

A market participant cannot lose money by trading, where $t(k)(t(l))$ to $T(k)(T(l))$ is the period experienced by a regular block.

The flexible block orders constraints:

$$
\sum \lambda_{i}^{d} \leq 1
$$

As with the flexible hourly order, a flexible block order can only win the bid once, where $\mathrm{i}(\mathrm{j})$ is the number of the regular blocks that belong to the same flexible block, and each regular block shall meet its own bidding constraints.

The extend linked block orders constraints:

$$
\lambda_{p} \geq \lambda_{c}
$$




$$
\begin{gathered}
\sum_{t=t(p)}^{T(p)} \lambda_{p}\left(p_{p}-\pi_{t}\right) q_{p, t}+\sum_{t=t(c)}^{T(c)} \lambda_{c}\left(p_{c}-\pi_{t}\right) q_{c, t} \geq 0 \\
\sum_{t=t(c)}^{T(c)} \lambda_{c}\left(p_{c}-\pi_{t}\right) q_{c, t} \geq 0
\end{gathered}
$$

Extend linked block order is more representative than linked block order. In this section, the bidding constraint of extend linked block orders is given directly, and the latter is ignored. In extend linked block, the child block must not lose money. If the child block is profitable, then the whole block will win the bid as long as the combination of the child and parent blocks is profitable. In the subscript of the above formula, $p$ represents the parent block and c represents the child block. Since the expression of the purchasing side and selling side is the same, the superscript $d$ and $g$ are omitted here and will not be repeated.

It is noted that the model is nonlinear caused by the multiplication of $0-1$ integer variables and continuous variables. In this case, there is a general auxiliary variable addition method that can transform it into a linear constraint:

$$
\begin{gathered}
-M x \leq y \leq M x \\
-M(1-x) \leq \pi-y \leq M(1-x)
\end{gathered}
$$

In the formula, $y$ is the added auxiliary continuous variable, $\mathrm{x}$ is a $0-1$ integer variable, $\pi$ is a continuous variable, and $\mathrm{M}$ is a sufficiently large positive number that needs to be greater than the upper limit of $\pi$. These two constraints are linear, and the effect of them is equivalent to the following equation:

$$
\mathrm{y}=\pi \mathrm{x}
$$

This allows a continuous variable to replace the product of a continuous variable with a $0-1$ variable, linearizing the model.

\subsection{Features}

Block orders are notable for their flexibility in that the same user can declare multiple types of blocks, that the same block can apply to multiple types of units, and that blocks are decoupled from each other. This can reasonably express the trading demands of market members with different physical and economic characteristics, and realize the simultaneous bidding of various types of energy and the orderly participation of multiple buyers in the market. For example, as shown in Fig. 1, thermal power units can use a combination of single hourly orders and regular block orders. The baseload part of the unit can be declared for the former to maintain the stable output of power, while the peak load part can be declared for regular block orders. This bidding way fully reflects the economic characteristics of the unit under different load rates. For thermal power units, it is useful to maximize its revenue, and for the system, it is helpful to peak shaving and valley filling, so as to alleviate the difficult problem of peak regulation. As shown in Fig. 2 , the BESS has greater freedom in the time of charge and discharge due to its high controllability. Therefore, under block orders mode, it can use flexible hourly orders or flexible block orders and make full use of the unit characteristics to benefit from peak shaving and valley filling.

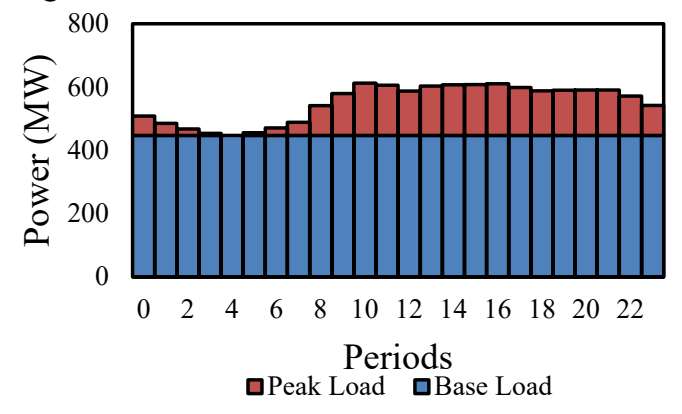

Fig. 1. Offering of thermal power unit.

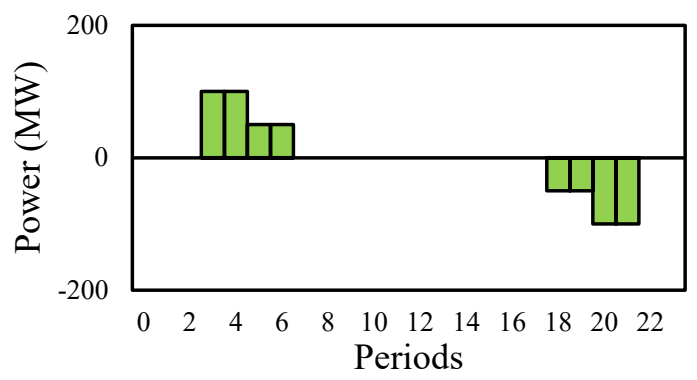

Fig. 2. Bidding of BESS.

In addition, block orders present strong compatibility and extensibility to be flexibly integrated with a variety of products. Especially, when new products are introduced to the market, it is easy to create new block types to meet requirements without redesigning rules.

The compatibility of Block Orders is shown in that block orders itself is just a bidding and clearing mechanism. After the market clearing is completed, each market participant gets the total bidding quantity and price of each period, and does not need to care about the bidding situation and constraint relationship of each specific order. In the subsequent settlement, energy deviation penalty and other links, only the power of participants in each period can be considered. Because the block orders mechanism generates time-sharing power, the curve separating is not required if the period of block order is the same length as the spot market period, or if the block orders period is longer than the spot market period, the curve breakdown is simpler.

Block orders is extensible in that its order type can be selected and added by the marketing operator. Therefore, it can push some order types as needed, such as the most basic order types: single hourly order, flexible hourly order, regular block, flexible block, and linked block. These basic order types already support trading well for most market participants. When the market is more perfect and the members are more mature, it can further introduce curtailable blocks, profiled blocks, etc., or design a new block that meets the needs. This is not difficult under the block orders mechanism. 


\section{Block orders implication for market participation models of BESS}

\subsection{Inspiration to the construction of Chinese electricity market}

In the future, the market reform inevitably requires the realization of the coordination of various energy sources and the introduction of diverse emerging users. At this point, block orders mode is very suitable to be introduced, because it is convenient for all types of units to bid on the same stage, and guide different types of loads to participate in the market in an orderly way, so as to meet the development requirements of the future market.

In addition, Block orders has two distinctive features that are consistent with the current state of the electricity market in China: compatibility and scalability. The significance of compatibility is reflected in the fact that many regional markets in China are not yet mature enough. On the one hand, it requires the introduction of new trading mechanisms to coordinate the trading demands of various members, and on the other hand, it is not suitable to make large-scale adjustments to the existing market rules. The significance of expansibility lies in that it is more consistent with the development law of the Chinese market to introduce the block orders mechanism step by step and design the order types that meet the actual needs of the market on this basis. The actual situation of Chinese market is impossible to be exactly the same as that of any other country in the world, and foreign rules cannot be completely copied. If necessary, existing rules should be innovated. The extensibility of the Block Orders mechanism makes this possible.

The block orders mechanism can be applied to the daily, weekly and monthly markets in China. In view of the fact that spot markets are usually centralized markets, Chinese application of block orders can be concentrated on monthly and weekly basis. One of the problems with building block orders in medium and long-term markets is the choice of period length. Generally speaking, the shorter the period, the greater the number of periods, the more the trading results can reflect the actual operation of the market, and the more accurate the price signal will be. But short period makes the bidding and the clearing more difficult; It is also difficult to guarantee the accuracy of load forecast and electricity price forecast at a time too early from the actual operation. Overall, a more appropriate choice is that in the weekly or shorter market, the length of the period is chosen as one hour; In the monthly market, the length is chosen to be four hours.

The block orders mechanism can be built and test from the monthly electricity market. In the early stage of market construction, only the following basic order types should be included: single hourly order, flexible hourly order, regular block, flexible block, and linked block. After the start of operation, we can collect all kinds of data and feedback opinions of market participants to further understand the operation of the mechanism. According to the actual needs of market participants and their familiarity with the mechanism, each type of block, such as curtailable blocks, profiled blocks and exclusive group, is added step by step to improve the existing block type or design new block type. In the initial stage, block orders monthly market will only be used for testing and will not actually affect the trading results. After the market construction is more mature and market participants are more familiar with the mechanism, it will be put into practice instead of the traditional monthly market.

After the monthly block orders market is established, a weekly market can be added. The weekly market uses a smaller time scales (one hour) to make the connection between different stages of the market more natural and smoother. The energy that market participants fail to trade in the monthly market or deviate due to changes in the actual situation can be traded in the weekly market, reducing the risk of directly bearing price fluctuations in the spot market. The participation of BESS in the weekly market is more flexible than that in the monthly market, and it is also more feasible in terms of policy than that in the spot market. Therefore, the construction of weekly block orders market is of great significance for BESS to participate in the medium and long-term market.

\subsection{Implications to medium and long-term trading model of BESS}

Block orders are suitable for various types of market participants, including, naturally, the BESS. In fact, the rapid growth of BESs is one of the important reasons for the adoption and development of block orders in Europe. BESS are characterized by limited energy storage, so they cannot be continuously charged or discharged over a long period of time. A typical operation of BESS is to charge to full capacity at a low price at the trough, sell the energy at a high price at other times or participate in the regulation market and lose energy due to repeated charges and discharges. In a normal market system, the closer to the actual running time, the greater the volatility of market prices, the more convenient it is for BESS to take advantage of their flexibility. The regulation market, on the other hand, is usually a spot market. Therefore, the main purpose of BESS to participate in the medium and long-term market is to obtain energy at a stable and low price, so as to ensure their ability to sell energy or participate in the regulation market. The establishment of block orders mechanism in the monthly and weekly market can effectively exert the flexibility of BESS, guarantee the energy acquisition, and promote the development of the BES industry.

The capacity/power ratio of the BESS widely used at present is usually 1 to 3 , that is, it takes 1 to 3 hours for the station to be fully charged from the zero energy. For BESS, the ideal situation is to find the one to three hours of each day with the lowest energy price and buy the energy during those hours to minimize charging costs. However, though rough trough periods can be obtained empirically, the exact trough periods are difficult to predict until the clearing is complete, especially in the medium-term market. Therefore, in the traditional medium and long-term market, BESS can only estimate the period of the lowest price and purchase energy, which 
suffers losses to a certain extent and fails to achieve the optimization of resource allocation.

In block orders, such problem is easy to solve, and BESS are particularly suited to submit flexible hourly orders and flexible block orders. In this way, they do not need to predict the lowest price period of each day by itself, but only needs to set the period of order as all possible periods of the lowest price. When the market clears, it will automatically find the period of the lowest price for the purchase. BESS can obtain energy stably in the medium-term market. Operators can also arrange bidding strategies in the spot market after the clearing results of the medium-term market are confirmed, such as participating in the regulation market or selling excess energy.

As the trading principles, block orders consider the commodity nature of electrical energy, minimizing the use of physical constraints and replacing them with block constraints. In this way, the coupling between periods will become weaker, which is very favorable for highly flexible BESS.

\section{Conclusions}

This paper analyzes the characteristics of block orders in foreign countries, points out its enlightenment to the construction of Chinese electricity market, puts forward the suggestions, and draws the following conclusions.

First, in order to adapt to the future development trend, block orders can be introduced into Chinese electricity market. Block orders with flexibility, compatibility, and scalability are suited to conditions of the market and help to build an energy Internet with coordination of centralization and decentralization and a wholesale and retail market with interaction of supply and demand in the future. The block orders mechanism can be built and test starting with the monthly market, introducing the base block types in batches and then introducing or creating the appropriate block types.

In addition, BESS are suitable for participating in the medium and long-term market, especially the weekly market, by means of flexible hourly or flexible block purchasing orders, to compensate for the energy loss caused by charging and discharging in the regulation market. Block orders can be set up in the monthly and weekly markets to solve the problem of BESS participating in the market. Thereafter, the time scale can be further narrowed to accommodate BES and market developments.

\section{References}

1. X. Zhang, L.J. Shi, Future Research Areas and Key Technologies of Electricity Market in China, Aut. Elec. Power Syst, 44(16), 1-11 (2020)

2. M IEC, Electrical energy storage white paper (IEC, Geneva, 2011)

3. D. Metz, Economic evaluation of energy storage systems and their impact on electricity markets in a smart-grid context (2017)
4. B.Q. Lin, W. Wu, Economic viability of battery energy storage and grid strategy: A special case of China electricity market, Energy, 124, 423-434 (2017)

5. Y.B. Jia, J. Zheng, H. Chen, et al, Capacity allocation optimization of energy storage in thermal-storage frequency regulation dispatch system based on EEMD, Power Syst. Technol, 42(9), 2930-2937 (2018)

6. J. Hu, S.L. Yang, C.Y. Hou, et al, Present condition analysis on typical demonstration application of large-scale energy storage technology and its enlightenment, Power Syst. Technol, 39(4), 879-885 (2015)

7. B. Dunn, H. Kamath, J.M. Tarascon, Electrical energy storage for the grid: a battery of choices, Sci, 334(6058), 928-935 (2011)

8. A.P. Roskilly, P.C. Taylor, J. Yan, Energy storage systems for a low carbon future-in need of an integrated approach, Appl. Energ, 137, 463-466 (2015)

9. P. Kanakasabapathy, Economic impact of pumped storage power plant on social welfare of electricity market, Int. J. Elec. Power Energ. Syst, 45(1), 187193 (2013)

10. M. Zidar, P.S. Georgilakis, N.D. Hatziargyriou, et al, Review of energy storage allocation in power distribution networks: applications, methods and future research, IET Gen. Transm. Distr, 10(3), 645652 (2016)

11. L. Ma, M.H. Fan, L. Guo et al, Latest development trends of international electricity markets and their enlightenment, Aut. Elec. Power Syst, 38(13), 1-9 (2014)

12. P. Zou, Q.X. Chen, Q. Xia, et al, Logical analysis of electricity spot market design in foreign countries and enlightenment and policy suggestions for China, Aut. Elec. Power Syst, 38(13), 18-27 (2014)

13. L. Ma, X.X. Zhang, Z. Li, et al, Enlightenment of Power Sales Side Market Liberalization in France, Sou. Power Syst. Technol, 9(8), 9-12 (2015)

14. Z. Li, B. Pang, G. Li, et al. Development of unified European electricity market and its implications for China, Aut. Elec. Power Syst, 41(24), 2-9 (2017)

15. NordPool, Day-ahead Market Regulations, $(2020 / 06 / 03)$

https://www.nordpoolgroup.com/globalassets/downl oad-center/rules-and-regulations/day-ahead-marketregulations_valid-from-3-june-2020.pdf

16. P.N. Biskas, D.I. Chatzigiannis, A.G. Bakirtzis, European electricity market integration with mixed market designs-Part I: Formulation, IEEE Tx. Power Syst, 29(1), 458-465 (2013)

17. M. Madani, M. Van Vyve, A new formulation of the European day-ahead electricity market problem and its algorithmic consequences, CORE discussion paper (2014) 
18. A. Martin, J.C. Müller, S. Pokutta, Strict linear prices in non-convex European day-ahead electricity markets, Opt. M. Softw, 29(1), 189-221 (2014)

19. L.M. Surhone, M.T. Tennoe, S.F. Henssonow, Cplex, (Betascript Publishing, Gütersloh, 2010)

20. C. Division, Using the Cplex callable library, $(2017 / 05 / 20)$

https://www.researchgate.net/publication/24367259

4_Using_The_Cplex_Callable_Library 